\title{
Large-eddy simulation of flow about a rotating cylinder at large Reynolds number
}

\author{
D. Pullin ${ }^{1}$, W. Cheng ${ }^{2}$ and R. Samtaney ${ }^{2}$ \\ ${ }^{1}$ Graduate Aerospace Laboratories, California Institute of Technology, Pasadena CA 91125, USA. \\ ${ }^{2}$ Mechanical Engineering, Physical Sciences and Engineering Division, King Abdullah University of Science and \\ Technology Thuwal, Saudi Arabia.
}

\begin{abstract}
Wall-resolved large-eddy simulations (LES) of flow past a rotating cylinder are described. The principal flow parameters are the dimensionless rotation speed $\alpha=\Omega D /\left(2 U_{\infty}\right)$ and the Reynolds number $R e_{D}=U_{\infty} D / \mathrm{v}$. We vary $R e_{D}$ at fixed $\alpha=0.6$ with emphasis on the lift-crisis phenomenon where, with increasing $R e_{D}$, the lift coefficient $C_{L}$ decreases suddenly as $R e_{D}$ passes though a narrow, critical band. Calculations of $C_{L}$ and $C_{D}$ are compared with experimental measurements to demonstrate that the lift crisis is captured by the LES. Results show that the mechanisms that drive the lift crisis when varying $R e_{D}$ at fixed $\alpha$ are similar to those observed when varying $\alpha$ at fixed $R e_{D}$. Both data sets support a hypothesis which interprets the lift crisis as the result of near-wall, small-scale reversal flows aggregating into a relatively narrow zone, producing small-scale incoherent separation accompanied by a deep pressure minimum. Scaling of the skin friction coefficient with $\operatorname{Re}_{D}^{1 / 2}$, which was found previously for flow past a non-rotating, is also observed for $\alpha=0.6$.
\end{abstract}

\section{Introduction}

The drag crisis for flow over a stationary, non-rotating cylinder is well known. This is characterized by a sudden decrease in the drag coefficient $C_{D}$ when the Reynolds number $\operatorname{Re}_{D}=U_{\infty} D / \mathrm{v}$ ( $D$ is the cylinder diameter, $U_{\infty}$ the free-stream speed and $v$ the kinematic viscosity) increases from subcritical to supercritical values over a range of approximately $\operatorname{Re}_{D}=2 \times 10^{5}-4 \times 10^{5}$. Lehmkuhl et al. [1] used LES to detect a mean-flow separation bubble upstream of the primary separation zone at supercritical $\operatorname{Re}_{D}$. The detailed, near-surface flow behavior through the drag crisis was studied by Cheng et al. [2], who observed local mean-flow separation bubbles at both sub-critical and supercritical $R e_{D}$. The azimuthal variation of the skin-friction coefficient obtained from their LES agreed well with experiment [3]. Cheng et al. concluded that the occurrence of the drag crisis was strongly related to the interaction of the main separation shear layer with unsteady, secondary flow cells that reattach within the large-scale separation zone. They argued that boundarylayer laminar to turbulent transition is more likely a strong effect of the drag crisis rather than its primary causal agent.

When the cylinder is rotating about its axis with constant rotational speed $\Omega$, the non-dimensional rotation speed $\alpha=$ $\Omega D /\left(2 U_{\infty}\right)$ is introduced as an additional flow parameter. That the lift coefficient $C_{L}$ generally increases with increasing $\alpha$ at fixed $R e_{D}$ was demonstrated in early experimental studies $[4,5]$. When $R e_{D}$ is fixed at values greater than about $R e_{D}=3.58 \times 10^{4}$, Swanson [6] found experimentally that $C_{L}$ suddenly decreased and subsequently continued to increase as $\alpha$ passed through a small band whose lower and upper bounding values were dependent on $\operatorname{Re}_{D}$. This has become known at the lift crisis. Subsequent experiments [7, 8] confirmed this effect. The lift crisis, also referred to as the inverse Magnus effect, has also been observed for flow over a rotating sphere where the axis of rotation is orthogonal to the direction of the free stream

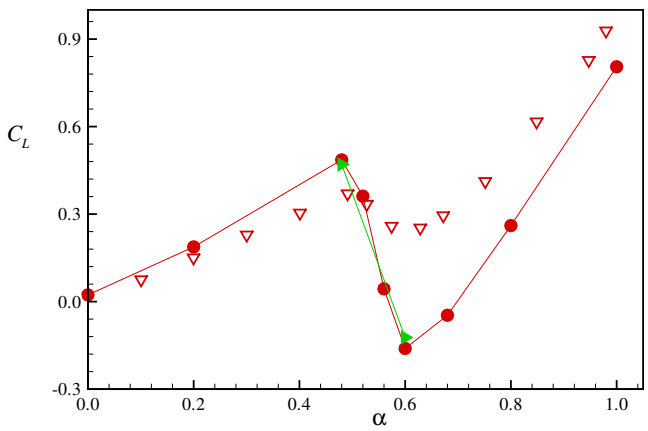

Figure 1: The lift crisis phenomenon viewed by varying $\alpha$ at fixed $\operatorname{Re}_{D}=6 \times 10^{4}$. Hollow symbols: experiment by Swanson [6]. Filled symbols: LES data.

\begin{tabular}{lllll}
\hline Case & $\operatorname{Re}_{D}$ & $N_{\theta}$ & $N_{r}$ & $N_{y}$ \\
\hline A1 & $5 \times 10^{3}$ & 512 & 256 & 96 \\
B1 & $1 \times 10^{4}$ & 512 & 256 & 96 \\
C1 & $2 \times 10^{4}$ & 512 & 256 & 96 \\
D1 & $4 \times 10^{4}$ & 2048 & 256 & 192 \\
E1 & $6 \times 10^{4}$ & 2048 & 256 & 192 \\
F1 & $8 \times 10^{4}$ & 2048 & 256 & 192 \\
G1 & $1 \times 10^{5}$ & 2048 & 256 & 192 \\
\hline
\end{tabular}

Table 1: Fixing $\alpha=0.6$ and varying $R e_{D}$.

[9].

Cheng et al. [10] implemented wall-resolved large-eddy simulation at two $R e_{D}$ with $\alpha$ in the range $0 \leq \alpha \leq 1.0$. At their low $R e_{D}=5 \times 10^{3}$, a lift crisis was not found. At $\operatorname{Re}_{D}=6 \times 10^{4}$, profiles of both pressure coefficient $C_{p}$ and skin-friction coefficient $C_{f \theta}$ clearly illustrate the presence of a lift crisis in $0.48 \leq \alpha \leq 0.6$. This range agrees well with experimental data [6]. Presently, we report results obtained from LES of flow over a cylinder with increasing $R e_{D}$ at $\alpha=0.6$ fixed. This will be shown to provide both an alternative view of the lift-crisis behavior, and will also give insight into the scaling of the azimuthal component of the surface skin-friction vector with $R e_{D}$ for a rotating cylinder flow.

\section{LES performed}

In Cartesian $(x, y, z)$ co-ordinates, we describe LES of flow in the $x$ direction about a cylinder rotating about the $y$-axis. The governing LES equations are solved numerically in time and space using a fourth-order accurate central-difference method where the skew-symmetric form of the convection term is discretized by an energy-conservative scheme. A standard fractional-step method is combined with a third-order RungeKutta method for time integration. A multigrid solver with linerelaxed Gauss-Seidel smoothing is utilized for solution of the 

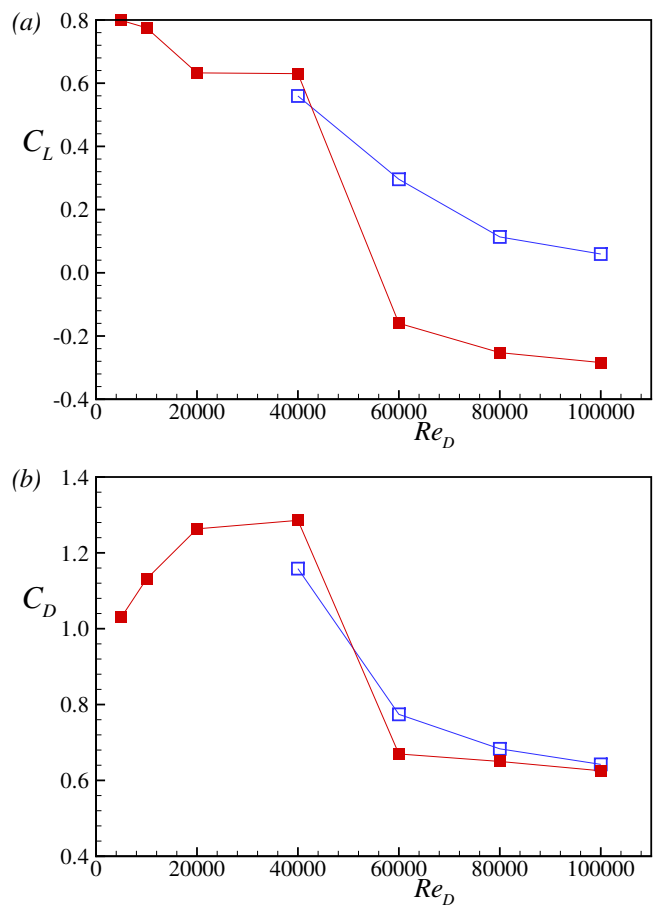

Figure 2: Fixing $\alpha=0.6$ and varying $\operatorname{Re}_{D}$. (a): $C_{L}$; (b): $C_{D}$. $\square$, experiments by [8]; $\mathbf{\square}$, present LES.

Helmholtz equations for velocity and the Poisson equations for pressure.

In the present LES, unresolved subgrid motions are represented by the stretched-vortex (SV) sub-grid scale model [11]. In cylindrical $(r, \theta, y)$ co-ordinates, the computational domain is a body-fitted O-grid with $L_{r}=40 D$ and $L_{y} / D=3.0$. Grid spacing is uniform in the $\theta$ and span-wise $y$-directions but is stretched in the $r$ direction. At the wall, a non-slip boundary condition is applied while the wall-normal grid resolution is of order the local viscous length scale $v / u_{\tau}$ where $u_{\tau}=\sqrt{\tau_{w} / \rho}$ is the local friction velocity and $\tau_{w}$ the local shear stress. Hence the LES is considered wall-resolved. The code has been verified in previous studies by comparison with DNS $[2,10]$.

The cylinder rotation is clockwise when viewed in the positive $y$ direction and the co-ordinate $\theta$ is taken as increasing clockwise with $\theta=0$ on the negative $x$-axis. The "top" of the cylinder is then at $\theta=90^{\circ}$, where the movement direction of the cylinder surface is parallel to the free stream while the "bottom" of the cylinder is $\theta=270^{\circ}$, where the cylinder surface moves antiparallel to the free stream. A range of LES were performed with different resolution $\left(N_{r}, N_{\theta}, N_{y}\right)$, as shown in table 1 where $R e_{D}=5 \times 10^{3}-10^{5}$. All flows have $\alpha=0.6$.

\section{Results}

Lift and drag coefficients $C_{L}, C_{D}$

Figure 1 shows LES results for the lift coefficient (in $z$ direction) $C_{L}(\alpha)$ versus $\alpha$ at fixed $R e_{D}=6 \times 10^{4}$ [10] . In $0.48 \leq \alpha \leq 0.6$, both the data of Swanson [6] and the LES show a lift crisis in the form of a sudden decrease of $C_{L}$ followed by recovery and subsequent increase. The local maximum $\rightarrow$ local minimum drop in $C_{L}$ given by the LES substantially exceeds that indicated by experiment. Swanson [6] does not give extensive details of the experimental technique. Experiments of flow past a rotating sphere [9] show a strong lift crisis with negative $C_{L}$ at comparable $\operatorname{Re}_{D}$ to the present LES.

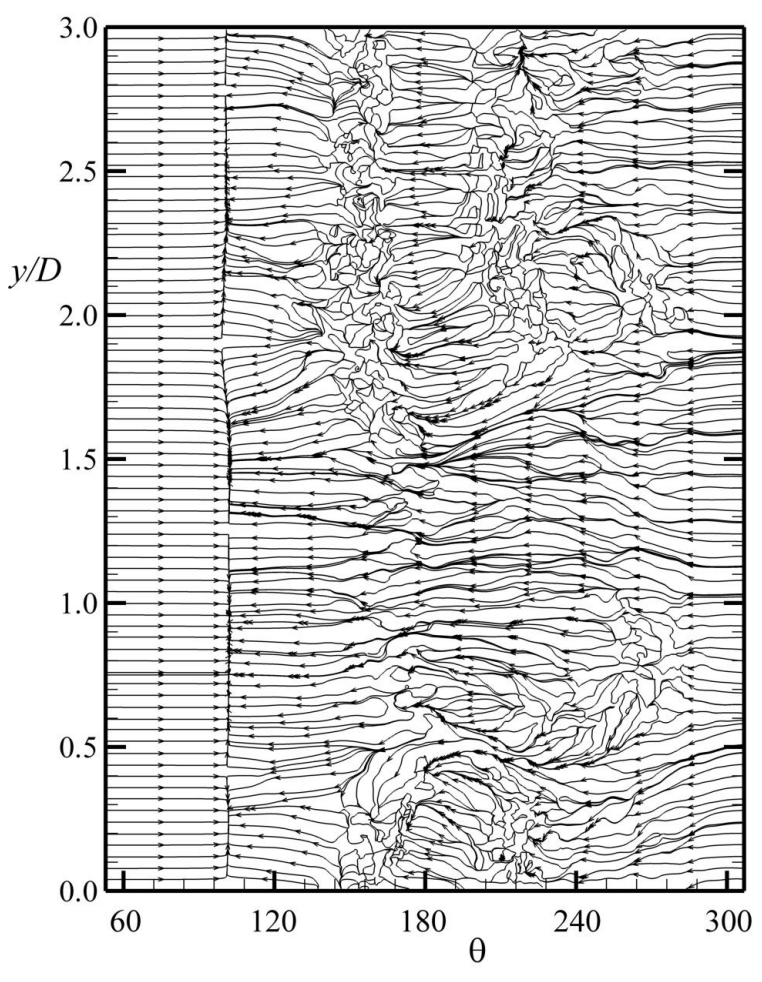

Figure 3: Skin friction lines at a time instant during a typical $C_{L}(t)$ cycle at $R e_{D}=4 \times 10^{4}, \alpha=0.6$ before the lift crisis.

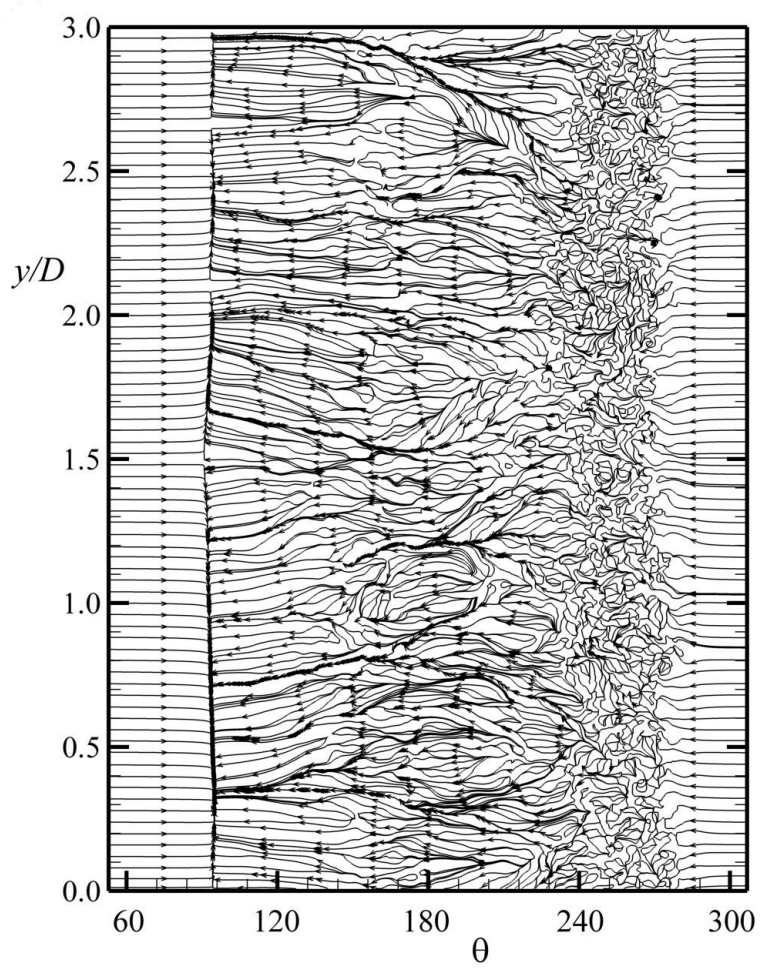

Figure 4: Skin friction lines at a time instant during a typical $C_{L}(t)$ cycle at $R e_{D}=6 \times 10^{4}, \alpha=0.6$ after the lift crisis. 
The variation of $C_{L}\left(R e_{D}\right)$ and $C_{D}\left(R e_{D}\right)$ with $R e_{D}$ for $\alpha=0.6$ are shown in figure 2 . In figure 2(a), the present LES shows a quite sharp decrease in $C_{L}$ over the range $4 \times 10^{4} \leq R e_{D} \leq$ $\times 10^{4}$. Experimental values [8] show a more moderate decline but over a similar range of $R e_{D}$. Both experiment and the present LES shows a substantial decrease in $C_{D}$ over $4 \times 10^{4} \leq$ $\operatorname{Re}_{D} \leq 6 \times 10^{4}$. This drag crisis, corresponding to a $50 \%$ reduction in $C_{D}$, is not as strong as that experienced by the nonrotating cylinder flow (at much larger $R e_{D}$ ).

\section{Cylinder surface skin-friction portraits}

To investigate the surface flow features before and after the lift crisis, we show plots of instantaneous skin-friction lines. These correspond to direction-field portraits of the surface skinfriction vector field $\mathbf{C}_{f}(\theta, y, t)$ on the developed cylinder surface. While the details of these plots show some variation during a typical vortex shedding or $C_{L}(t)$ cycle at any given $\left(R e_{D}, \alpha\right)$, structural changes in general features of the portraits as $R e_{D}$ is increased at fixed $\alpha$, can provide insight into flow separation events through the lift crisis. Figure 3 shows a portion in $\left(55^{\circ} \leq \theta \leq 305^{\circ}, 0 \leq y / D \leq 3.0\right)$ of a typical instantaneous $\mathbf{C}_{f}(\theta, y, t=$ const $)$ image at $R e_{D}=4 \times 10^{4}, \alpha=0.6$ prior to the lift crisis. The separatrix corresponding to the primary shear layer separation is clearly visible at $\theta \approx 95-100^{\circ}$. At larger $\theta$ patches of scattered, small-scale separation/reattachment cells can be see as indicated by the presence of critical points of the $\mathbf{C}_{f}$ field. These events co-exist with regions of coherent, nearly uniform flow in the anticlockwise direction. This mixing of local separation and attached, near-surface back flow is sufficiently strong to form both wall-attached and fully-separated vortices [10]

When $R e_{D}=6 \times 10^{4}, \alpha=0.6$ after to the lift crisis, figure 4 shows a transition to a more ordered state where the smallscale separation/reattachment cells have aggregated into a narrow band in $230^{\circ} \leq \theta \leq 270^{\circ}$ that is coherent across the whole span. This indicates the presence of strong, highly localized near-surface vorticity associated with the formation of a local deep pressure minimum (see figure 5). Apart from primary separation on the top cylinder surface, the flow is largely attached elsewhere. This sudden transition in surface separation patterns is the dynamical signature of the list crisis. We have shown that it is invariant when viewed either at fixed $\operatorname{Re}_{D}$ with increasing $\alpha$ [10] or conversely with fixed $\alpha$ with increasing $R e_{D}$.

\section{Pressure coefficient $C_{p}$ and skin friction coefficient $C_{f \theta}$}

Distributions of span-wise and time-averaged $C_{p}(\theta)$ are shown in figure 5. The variation of $C_{p}(\theta)$ is similar for both $R e_{D}=$ $5 \times 10^{3}, 10^{4}$ (figure 5(a)) including a minimum region around the top side of the cylinder and a plateau value of $C_{p} \approx-1$ on the rear or leeward side of the cylinder. At higher $\operatorname{Re}_{D}=$ $2 \times 10^{4}, 4 \times 10^{4}$, the plateau decreases to about $C_{p} \approx-1.3$. Further increase in $R e_{D}$ leads to substantial changes in $C_{p}(\theta)$. For $R e_{D}=6 \times 10^{4}$, shown in both figures $5(\mathrm{a})$ and $5(\mathrm{~b})$, the minimal $C_{p}$ on the top side of the cylinder is increased markedly while the plateau region is shifted to about $C_{p} \approx-0.7$. On the bottom side of the cylinder, where the cylinder motion opposes the free-stream velocity, a sharp decrease in the $C_{p}$ minimum at about $\theta=270^{\circ}$ can be observed. This change gives rise to the lift crisis. As shown in figure 5(b), further increase in $R e_{D}$ does not produce substantial changes in $C_{p}(\theta)$ distributions.

Corresponding distributions of the azimuthal coefficient of the skin-friction $C_{f \theta}$ are shown in figure 6 . The subfigures $6(\mathrm{a}) / 6(\mathrm{~b})$ show $R e_{D}$ below/above the lift crisis, respectively, except that $R e_{D}=6 \times 10^{4}$ is shown in both plots. For these distributions, in addition to the monotonic decrease of the peak $C_{f \theta}$
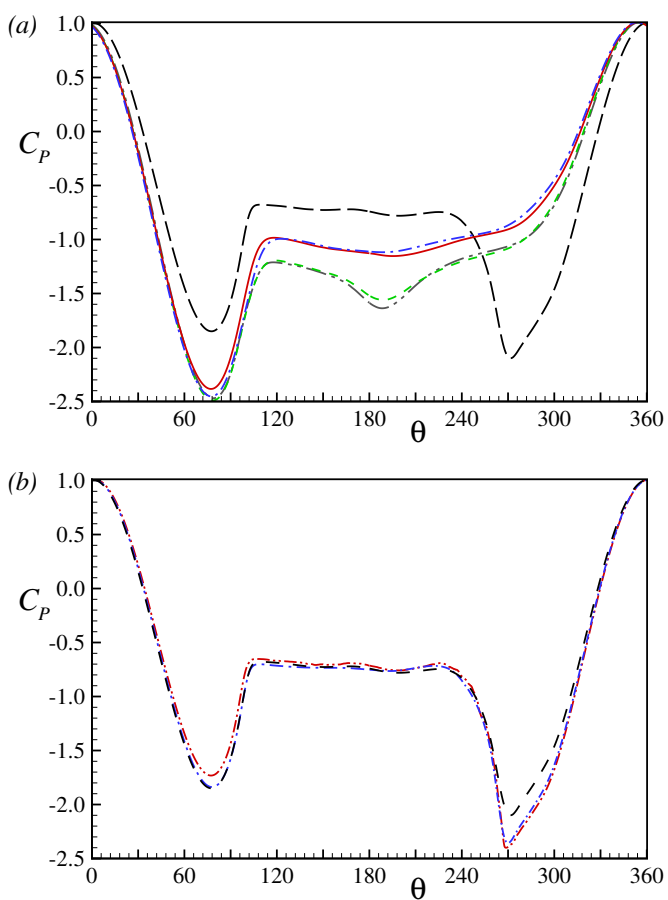

Figure 5: $\alpha=0.6$. Pressure coefficient $C_{p}(\theta)$. (a): - - $\operatorname{Re}_{D}=5 \times 10^{3} ;-, \operatorname{Re}_{D}=1 \times 10^{4} ;---, \operatorname{Re}_{D}=2 \times$ $10^{4} ;-\cdots-, \operatorname{Re}_{D}=4 \times 10^{4} ;----, \operatorname{Re}_{D}=6 \times 10^{4}$. (b): ,$---- \operatorname{Re}_{D}=6 \times 10^{4} ;-\cdot-, \operatorname{Re}_{D}=8 \times 10^{4}$; $R e_{D}=1 \times 10^{5}$.
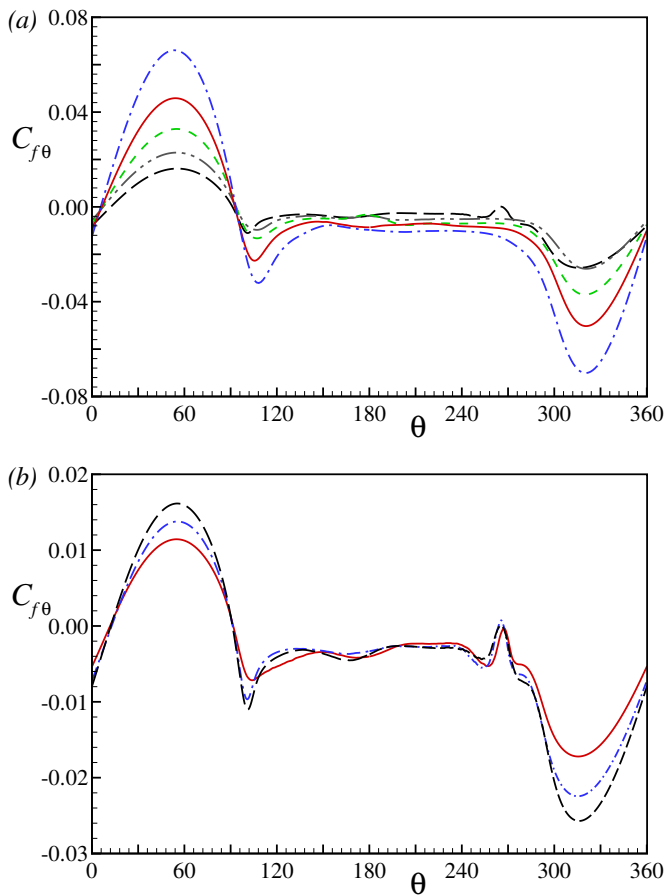

Figure 6: $\alpha=0.6$. Skin friction coefficient $C_{f \theta}(\theta)$. (a): - - $\operatorname{Re}_{D}=5 \times 10^{3} ;-, \operatorname{Re}_{D}=1 \times 10^{4} ;----, \operatorname{Re}_{D}=2 \times$ $10^{4} ;-\cdots-, \operatorname{Re}_{D}=4 \times 10^{4} ;----, \operatorname{Re}_{D}=6 \times 10^{4}$. (b): ,$---- \operatorname{Re}_{D}=6 \times 10^{4} ;-\cdot-, \operatorname{Re}_{D}=8 \times 10^{4}$; $\operatorname{Re}_{D}=1 \times 10^{5}$. 

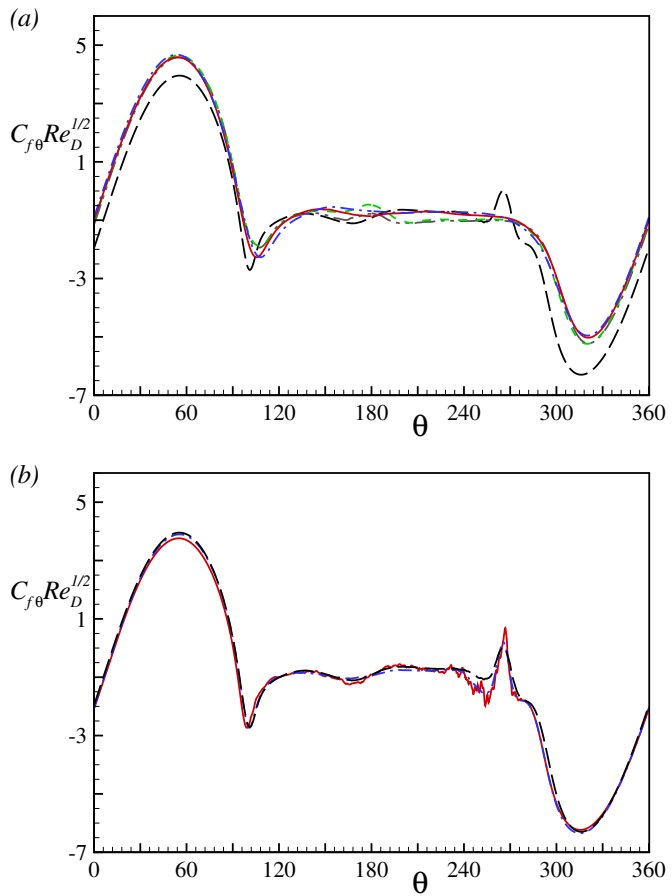

Figure 7: $\alpha=0.6$. Scaled skin friction coefficient. (a): - - $R e_{D}=5 \times 10^{3} ; \longrightarrow, \operatorname{Re}_{D}=1 \times 10^{4} ;----, \operatorname{Re}_{D}=2 \times$ $10^{4} ;-\cdots-, R e_{D}=4 \times 10^{4} ;----, R e_{D}=6 \times 10^{4}$. (b): ,$---- \operatorname{Re}_{D}=6 \times 10^{4} ;-\cdot-, \operatorname{Re}_{D}=8 \times 10^{4} ;-$ $R e_{D}=1 \times 10^{5}$.

at around $\theta=60$ with increasing $R e_{D}$, the main effect of $R e_{D}$ is the appearance of a small local maximum or "hill" at about $\theta=270^{\circ}$. This is absent for $R e_{D} \leq 4 \times 10^{4}$, but is observed for $R e \geq 6 \times 10^{4}$.

For over a cylinder with $\alpha=0$ Cheng et al. [2] found evidence that the scaled skin-friction $C_{f \theta}(\theta) R e_{D}^{1 / 2}$ showed good collapse independent of $\operatorname{Re}_{D}$ with a peak value $C_{f \theta} R e_{D}^{1 / 2} \approx 5$. In figure 7, we show $C_{f \theta}(\theta) R e_{D}^{1 / 2}$ for varying $R e_{D}$ with $\alpha=0.6$. Here also, subfigures 7(a)/7(b) show distributions for $R e_{D}$ below/above the lift crisis, respectively, except that $R e_{D}=6 \times 10^{4}$ is common to both plots.

It is evident that, for the range of $\operatorname{Re}_{D}$ shown, there is good collapse of $C_{f \theta}(\theta) \operatorname{Re}_{D}^{1 / 2}$ for both subcritical and for super-critical $R e_{D}$, but that the collapse lines are rather different for the two $R e_{D}$ ranges. For $R e_{D} \leq 4 \times 10^{4}$, the peak scaled skin friction on the top side of the cylinder reaches $C_{f \theta} R e_{D}^{1 / 2} \approx 4.8$ while on the bottom side we find a minimum $C_{f \theta} R e_{D}^{1 / 2} \approx-5$. For $R e_{D} \geq 6 \times 10^{4}$, the peak on the top side decreases to about $C_{f \theta} R e_{D}^{1 / 2} \approx 4$ and the corresponding bottom side decreases to about $C_{f \theta} R e_{D}^{1 / 2} \approx-6.5$. We conclude that at constant $\alpha=0.6$, the scaling $C_{f \theta}(\theta) \operatorname{Re}_{D}^{1 / 2}$ remains valid, but separate, over subcritical and supercritical $R e_{D}$.

\section{Conclusion}

Large-eddy simulation has been used to investigate the lift crisis phenomenon in high Reynolds number rotating cylinder flows. With $\alpha=0.6$, our LES captures a decrease of $C_{L}$ from $\operatorname{Re}_{D}=4 \times 10^{4}$ to $6 \times 10^{4}$. Instantaneous skin friction lines are used to reveal changes in flow separation that occur as $R e_{D}$ passes through the lift crisis. Changes in the surface skinfriction portraits are found to be essentially the same as those observed when the lift crisis is viewed at fixed $\alpha$ but with increasing $R e_{D}$. The common underlying mechanism is characterized by a change in surface-separation structure, on the leeward and bottom cylinder surface, from scattered separation cells to near-wall, small-scale reversal flows aggregating into a relatively narrow azimuthal zone. This produces both small-scale incoherent separation and a strong pressure minimum. The scaling of the skin friction as $C_{f \theta}(\theta) \operatorname{Re}_{D}^{1 / 2}$ is found to work quite well at $\alpha=0.6$.

\section{Acknowledgement}

The Cray XC40 Shaheen at KAUST was used for all simulations reported.

\section{References}

[1] O. Lehmkuhl, I. Rodriguez, R. Borrell, J. Chiva and A. Oliva. Unsteady forces on a circular cylinder at critical Reynolds numbers. Phys. Fluids 26, 4904415, 2014.

[2] W. Cheng, D.I Pullin, R. Samtaney, W. Zhang, and W. Gao. Large-eddy simulation of flow over a cylinder with $\operatorname{Re}_{D}$ from $3.9 \times 10^{3}$ to $8.5 \times 10^{5}$ : a skin-friction perspective. J. Fluid Mech. 820, 121-158, 2017.

[3] E. Achenbach. Distribuion of local pressure and skin friction around a circular cylinder in cross-flow up to $\mathrm{Re}=5 \times$ $10^{6}$. J. Fluid Mech. 34, 625-639, 1968

[4] E.G Reid. Tests of rotating cylinders. Tech. Rep. No. 209. National Advisory Committee for Aeronautics, 1924.

[5] A Thom. Experiments on the flow past a rotating cylinder. Aeronautical Research Committee, Reports and Memoranda 1410, 1931.

[6] W.M. Swanson The Magnus effect: A summary of investigations to date. Journal of Basic Engineering 83, 461-470, 1961.

[7] C.A Fletcher. Negative Magnus forces in the critical Reynolds number regime. J. Aircraft 9, 826-834, 1972.

[8] S. Takayama, and K Aoki. Flow characteristics around a rotating grooved circular cylinder with grooved of different depths. Journal of Visualization 8, 295-303, 2005.

[9] J. Kim., H. Choi, H. Park and J.Y. Yoo Inverse Magnus effect on a rotating sphere: when and why, J. Fluid Mech. 754, R2, 2014

[10] W. Cheng., D. Pullin, R. Samtaney Large-eddy simulation of flow over a rotating cylinder: the lift crisis at $\operatorname{Re}_{D}=6 \times 10^{4}$, J. Fluid Mech. 855, 371-407, 2018

[11] A. Misra, A. and D. I. Pullin. A vortex-based subgrid stress model for large-eddy simulation. Phys. Fluids $\mathbf{9}$, 2443-2454, 1997 